\title{
Apolipoprotein A-1 regulates osteoblast and lipoblast precursor cells in mice
}

\author{
Harry C Blair ${ }^{1,2}$, Elena Kalyvioti ${ }^{3}$, Nicholaos I Papachristou ${ }^{3}$, Irina L Tourkova ${ }^{1,2}$, Spryros A Syggelos ${ }^{3}$, Despina Deligianni ${ }^{4}$, \\ Malvina G Orkoula ${ }^{5}$, Christos G Kontoyannis ${ }^{5,6}$, Eleni A Karavia7, Kyriakos E Kypreos ${ }^{7}$ and Dionysios J Papachristou ${ }^{2,3}$
}

Imbalances in lipid metabolism affect bone homeostasis, altering bone mass and quality. A link between bone mass and high-density lipoprotein (HDL) has been proposed. Indeed, it has been recently shown that absence of the HDL receptor scavenger receptor class B type I (SR-B1) causes dense bone mediated by increased adrenocorticotropic hormone (ACTH). In the present study we aimed at further expanding the current knowledge as regards the fascinating bone-HDL connection studying bone turnover in apoA-1-deficient mice. Interestingly, we found that bone mass was greatly reduced in the apoA-1-deficient mice compared with their wild-type counterparts. More specifically, static and dynamic histomorphometry showed that the reduced bone mass in apoA-1 ${ }^{-/-}$mice reflect decreased bone formation. Biochemical composition and biomechanical properties of $A p o A-1^{-1-}$ femora were significantly impaired. Mesenchymal stem cell (MSC) differentiation from the apoA-1 $1^{-/-}$mice showed reduced osteoblasts, and increased adipocytes, relative to wild type, in identical differentiation conditions. This suggests a shift in MSC subtypes toward adipocyte precursors, a result that is in line with our finding of increased bone marrow adiposity in apoA-1 $1^{-1-}$ mouse femora. Notably, osteoclast differentiation in vitro and osteoclast surface in vivo were unaffected in the knock-out mice. In whole bone marrow, PPAR $y$ was greatly increased, consistent with increased adipocytes and committed precursors. Further, in the apoA-1 $1^{-1-}$ mice marrow, CXCL12 and ANXA2 levels were significantly decreased, whereas CXCR4 were increased, consistent with reduced signaling in a pathway that supports MSC homing and osteoblast generation. In keeping, in the apoA-1 $1^{-/-}$animals the osteoblast-related factors Runx2, osterix, and Col1a1 were also decreased. The apoA-1 ${ }^{-1-}$ phenotype also included augmented CEPBa levels, suggesting complex changes in growth and differentiation that deserve further investigation. We conclude that the apoA-1 deficiency generates changes in the bone cell precursor population that increase adipoblast, and decrease osteoblast production resulting in reduced bone mass and impaired bone quality in mice.

Laboratory Investigation (2016) 96, 763-772; doi:10.1038/labinvest.2016.51; published online 18 April 2016

Osteoporosis is characterized by low bone mass and microarchitectural deterioration of bone, resulting in bone fragility and fracture susceptibility. ${ }^{1,2}$ It is a major public health problem worldwide; indeed, in the United States, more than 44 million people have osteoporosis or low bone mass. ${ }^{3}$ The pathogenesis of osteoporosis is multifactorial. Age, sex, body size, metabolic factors, and genetic susceptibility are contributory factors. Interestingly, several studies have shown that there are strong links between bone, fat metabolism, and systemic energy metabolism, and that perturbations in lipid regulatory pathways may trigger both local and systemic phenomena that ultimately affect osteoblast and/or osteoclast function, resulting in osteoporosis. ${ }^{4-11}$ It is wellknown that mesenchymal stem cells (MSC) have the capacity to differentiate toward lipoblasts or osteoblasts and that differentiation of MSCs to one lineage suppresses the other. The fate of MSC depends upon the activation of specific signaling transduction pathways, namely peroxisome proliferator-activated receptor- $\gamma$ (PPAR- $\gamma$ ), bone morphogenetic proteins (BMPs), and the Wnt signaling pathway that differentially regulates adipogenic or osteogenic differentiation. ${ }^{9}$

Several workers have studied the implication of several lipid and lipoprotein regulators in the pathobiology of

${ }^{1}$ Pittsburgh VA Medical Center, Pittsburgh, PA, USA; ${ }^{2}$ Department of Pathology, University of Pittsburgh, Pittsburgh, PA, USA; ${ }^{3}$ Department of Anatomy-HistologyEmbryology, Unit of Bone and Soft Tissue Studies, University of Patras School of Medicine, Patras, Greece; ${ }^{4}$ Department of Mechanical Engineering and Aeronautics, University of Patras, Patras, Greece; ${ }^{5}$ Department of Pharmacy, University of Patras, Patras, Greece; ${ }^{6}$ Institute of Chemical Engineering Sciences, Foundation for Research and Technology-Hellas (FORTH/ICE-HT), Patras, Greece and ${ }^{7}$ Department of Pharmacology, University of Patras Medical School, Patras, Greece

Correspondence: Professor DJ Papachristou, MD, PhD, Department of Anatomy-Histology-Embryology, Unit of Bone and Soft Tissue Studies, University of Patras School of Medicine, University Campus, Building of Preclinical Studies, 2nd Floor, Room B48, Patras 26504, Greece.

E-mail: papachristoudj@med.upatras.gr or dip13@pitt.edu

Received 29 December 2015; revised 11 February 2016; accepted 18 March 2016 
osteoporosis. In this vein it has been recently documented that apolipoprotein $\mathrm{E}$ is a regulator of bone mass and turnover, both in vitro and in vivo. ${ }^{12-14}$ Moreover, epidemiological data propose that low levels of high-density lipoprotein cholesterol (HDL-C) are linked to osteoporosis; ${ }^{15}$ nevertheless, the mechanism that underlines this association is still unclear. Further adding to the complexity of bone-fat connection, it has been recently shown that a defect in the HDL receptor scavenger receptor class B type I (SR-B1) causes osteosclerosis, but by an unrelated mechanism, traced to HDL cholesterol delivery for cortisol production in the adrenal with consequent increases in adrenocorticotropic hormone (ACTH) that compensate and normalize cortisol, rather than a direct effect on bone production. ${ }^{16,17} \mathrm{ACTH}$ is, in turn, an osteoblast growth factor and inducer of vascular endothelial growth factor in bone. ${ }^{18}$

The production of HDL requires the major cholesterol binding protein, apolipoprotein A-1 (ApoA-1), a lipid transporter, ATP-binding cassette 1 and the plasma enzyme lecithin:cholesterol acyl tranferase (LCAT). A second apolipoprotein, ApoA-2, is normally about $20 \%$ of the lipoprotein component. ApoA-1, is a 243 -amino acid protein, which is synthesized mainly in the liver and intestine. Through a series of intermediate steps, minimally lipidated apoA-1 forms discoidal HDL particles, is converted to spherical particles by LCAT, and is eventually cleared via SR-B1. ${ }^{19-21}$ ApoA-1 is critical for the formation and final size of the HDL particles. ${ }^{22}$ Our research group has studied the role of HDL metabolic pathway in the pathogenesis of several diseases including osteoarthritis. ${ }^{23-25}$ In fact, we have recently shown that apoA-1- and LCAT-deficient mice develop osteoarthritis when stressed with high-fat diet. ${ }^{25}$

Triggered by the current knowledge and our research data in the present study we examined whether apoA-1 deficiency, and thus severely impaired HDL synthesis, affects the differentiation of bone marrow MSC in vitro and/or bone quality in vivo. Interestingly, we uncovered that the ApoA-1deficient mice are severely osteoporotic with a complex phenotype that implicates HDL as a requirement for normal cell-fate selection in MSC in bone.

\section{MATERIALS AND METHODS}

\section{Experimental Animal Models}

Male 12-week-old ApoA-1-deficient male mice (ApoA-1 ${ }^{-1-}$ ), backcrossed on C57BL/6 10 generations, and wild-type C57BL/6 (WT) mice (Jackson Labs, Bar Harbor, ME, USA) were maintained on standard chow diet, $29 \%$ protein, $60 \%$ carbohydrates, $11 \%$ fat (Mucedola SRL, Milan Italy), ad libitum in a $12 \mathrm{~h}$ dark/light cycle (0700 hours to 1900 hours light). Animals were genotyped by tail DNA PCR. Groups of mice had similar average body weight, plasma cholesterol, triglycerides, and glucose. Mice were caged individually. At 12 weeks, mice were killed and lumbar vertebrae and femora were collected for analyses in accord with published standards. ${ }^{26}$ Animal studies were conducted according to the EU guidelines for the Protection and Welfare of Animals. The estimated sample size was determined by power analysis, using the tool (http://www.stat.ubc.ca/ rollin/stats/ssize/n2. html). All animal studies were performed at least three times. Replicates refer to independent biological data points. No data were excluded. The work was authorized by the committee of the Laboratory Animal Center of The University of Patras Medical School and the Veterinary Authority of the Prefecture of Western Greece.

\section{Static and Dynamic Histomorphometry}

Seven and 2 days before killing, mice were injected calcein $(25 \mu \mathrm{g} / \mathrm{g})$, intra-peritoneally. For histology and microcomputed tomography, specimens were analyzed in a randomized and blinded fashion. Briefly, femora and lumbar vertebrae were fixed in $4 \%$ formalin overnight at $4{ }^{\circ} \mathrm{C}$ and stored at $70 \%$ ethanol at $-20^{\circ} \mathrm{C}$ until used. Fixed samples were studied by micro-CT Scanner (VivaCT40, Scanco Medical AG). For dynamic histomorphometry, undecalcified frozen bone tissues were sectioned at $4 \mu \mathrm{m}$ on a Microm HM 505E cryostat using tape transfer (CryoJane Instrumetics, St. Luis, MO, USA). In vivo osteoblast and osteoclast parameters were generated from frozen sections of decalcified femora. Fluorochrome measurements used a Nikon TE2000 inverted phase-\$fluorescence microscope with a 12 bit $1600 \times 1200$ pixel CCD (Spot, Sterling Heights, MI, USA). Tartrate-resistant acid phosphatase (TRAP) activity was visualized using $0.01 \%$ naphthol phosphate substrate (Sigma, St Louis, MO, USA) in N-N-dimethylformamide (Sigma) followed by the addition of $5 \mathrm{mg} / \mathrm{ml}$ fast red violet in $50 \mathrm{mM}$ sodium tartrate and $90 \mathrm{mM}$ sodium acetate at $\mathrm{pH}$ 5.0. Histological studies were performed by observers blinded to groups. Measurements used published standards. ${ }^{26}$

\section{MSC Isolation and Osteoclast Differentiation}

For MSC preparation and differentiation, femora were removed and whole bone marrow cells were flushed with RPMI-1640 plus 10\% FBS (Gibco, Grand Island, NY, USA) and plated at $2 \times 10^{5} / \mathrm{cm}^{2}$ at $37^{\circ} \mathrm{C}$, in $\alpha$-MEM, $10 \%$ FBS and antibiotics (Gibco). At $48 \mathrm{~h}$, non-adherent cells were removed; adherent cells were cultured in in the same medium for expansion. Media were changed every 3 days. Cultures were passaged at $80 \%$ confluence and replated at $5 \times 10^{5}$ cells/ $\mathrm{cm}^{2}$. For differentiation used special media for 21 days. Adipogenic medium was growth medium plus $10 \mu \mathrm{g} / \mathrm{ml}$ insulin, $0.5 \mathrm{mM}$ isobutylmethylxanthine, $10 \mathrm{nM}$ dexamethasone, $0.2 \mathrm{mM}$ indomethacin, $10 \mu \mathrm{M}$ rosiglitazone, and $0.2 \mathrm{nM}$ L-triiodothyronine. Osteogenic medium was growth medium with L-ascorbic acid, $50 \mu \mathrm{g} / \mathrm{ml}, 100 \mathrm{mM} \beta$-glycerolphosphate, and $10 \mathrm{nM}$ dexamethasone (Sigma). The media were replaced every 3 days. Alkaline phosphatase (ALP) activity used $0.05 \%$ Napthol AS-MX phosphate substrate in 2.8\% N,N-dimethyl formamide and $0.1 \mathrm{M}$ Tris maleate, $\mathrm{pH} 8.4$ with $0.1 \%$ fast blue to visualize the product (Sigma). Mineral was visualized in $1 \%$ silver nitrate exposed to strong light (von Kossa). 
Adipocytes were stained using $0.2 \%$ Oil Red $\mathrm{O}$ in $60 \%$ isopropanol. Digital images of different areas of each dish were obtained (original magnification $\times 10$ ) and imported into Image J (Java-based image processing program, NIH) for densitometry analysis.

For osteoclast differentiation, $2 \times 10^{5}$ cells $/ \mathrm{cm}^{2}$ bone marrow cells were cultured with $\alpha$-MEM, 10\% FBS with $30 \mathrm{ng} / \mathrm{ml}$ murine M-CSF (R\&D Systems, Abingdon UK) and $10 \mathrm{ng} / \mathrm{ml}$ murine RANKL (PeproTech, Rocky Hill, NJ, USA) for 14 days.

\section{Bone Quality Studies}

Bone quality studies included Raman spectra, acquired focusing a $785 \mathrm{~nm}$ laser through an objective lens $(\times 20)$ onto femora using an inVia Raman microscope (Renishaw, UK). Raman emission from 700 to 1800 per $\mathrm{cm}$ was scanned. Spectra were collected from the periosteal surface of the entire diaphysis. The amide I (1590-1710 per $\mathrm{cm})$ region in the spectrum was isolated for further analysis. Sub-bands were derived by the second-derivative method. Deconvolution and curve fitting of the sub-bands to unconstrained GaussianLorentzian profiles used Peakfit 4.0 (Jandel, San Rafael, CA, USA). The ratio of $1668: 1690$ per $\mathrm{cm}$ was calculated to evaluate the cross-linking and quality of collagen. ${ }^{27}$ Threepoint bending was done with a MiniMat2000 (Rheometric Scientific, Piscataway NJ, USA) with a $12 \mathrm{~mm}$ distance between supports. Load-deformation curves and maximal load recorded at a $2 \mathrm{~mm} / \mathrm{min}$ bending rate. Hue, saturation, and intensity threshold mapping was used to quantify adipocyte and non-adipocyte areas in cross paraffin sections of mice femora (Fovea Pro, Reindeer Graphics, Ashville, NC, USA).

\section{In vitro Studies}

For Western blots, MSCs were lysed in 0.1\% SDS, $1 \%$ octylphenoxypolyethoxyethanol, $1 \%$ sodium deoxycholate, $150 \mathrm{mM} \mathrm{NaCl}, 0.5 \mathrm{mM}$ EDTA, and $10 \mathrm{mM}$ Tris, pH 7.5 with a proteinase and phosphatase inhibitors (Sigma). Blot was probed using PPAR $\gamma$ antibody sc7273, a mouse monoclonal specific to amino acids 480-505 at the C-terminus (Santa Cruz, Santa Cruz, CA, USA) or RUNX2 antibody sc10758, a rabbit polyclonal IgG raised to amino acids 294-363, an internal site in the protein (Santa Cruz). Incubation was $1 \mathrm{~h}$ at room temperature and then $1 \mathrm{~h}$ with secondary antibody conjugated to horseradish peroxidase (goat anti-mouse IgGHRP, sc-2005, Santa Cruz or goat anti-rabbit IgG-HRP, AP132P, Millipore, Billerica, MA, USA). Antibody binding was visualized using by enhanced chemiluminescence (SuperSignal, ThermoScientific, Rockford, IL, USA). The results are expressed in relation to actin mouse monoclonal antibody MAB1501 (Millipore).

For flow analysis, cells were fixed and permeabilized for 20 min at $4{ }^{\circ} \mathrm{C}$ (Cytofix-Cytoperm, BD Biosciences, Franklin Lakes, NJ, USA). Cells were labeled with the primary antibodies used for Western blots for $1 \mathrm{~h}$ at $4{ }^{\circ} \mathrm{C}$. Cells were then incubated with secondary antibodies conjugated with Alexa Fluor 488 for $1 \mathrm{~h}$ at $4{ }^{\circ} \mathrm{C}$ and then subjected to flow cytometric analysis on a EPICS-XL/Coulter instrument and analyzed using FlowJo software (Tree Star, Ashland, OR, USA).

RNA extraction, reverse transcription, and PCR were essentially as reported. ${ }^{16}$ The threshold amplification cycles was converted to relative expression as previously described. ${ }^{16}$ Specificity of the products was confirmed by agarose electrophoresis. Primer sets are shown in Supplementary Table S2.

All in vitro experiments were repeated three times.

\section{Blood Analyses}

Serum was separated by centrifugation of clotted blood. ACTH and cortisol were assayed on the Immulite 2000 (Siemens USA Healthcare, Malvern, PA, USA) using clinical assay standards and procedures.

\section{Statistical Analyses}

Statistical comparisons used Student's $t$-test. Data are shown as mean \pm standard deviation (mean \pm s.d.). Differences were considered significant at $P \leq 0.05$. All analyses were performed using GraphPad Prism (version 5.03, GraphPad Software, San Diego, CA, USA).

\section{RESULTS}

\section{Apoa-1 Deficiency Results in Reduced Bone Mass}

Changes in bone density were measured by micro-computed tomography of mice lumbar vertebral bodies, which consist primarily of trabecular bone. Bone volume by all measures, including trabecular thickness, was markedly and significantly decreased, by a factor of about 3 , in the apoA-1-deficient mice compared with their control littermates. The appearance of the vertebrae on $\mu \mathrm{CT}$ is shown (Figure 1a) and major static parameters (Figure 1b). Dynamic histomorphometry on decalcified sections from lumbar vertebrae showed that apoA-1-deficient mice displayed significantly decreased calcein-labeled, and double-labeled surfaces, as well as significantly reduced bone formation rate; calcein interlabel distance was similar between the two groups (Figures $1 \mathrm{c}$ and d); comprehensive parameters are given in Supplementary Table S1. ApoA-1 deficiency did not affect osteoclastic function; the presence of osteoclasts per bone area as determined by TRAP labeling was similar between the two groups (Figures 1c and d).

Biochemical composition and bone strength were also studied. Raman spectroscopy showed the amide I envelope, corresponding to collagen secondary structure, was altered in the knock-out compared with the WT mice. Specifically, there was a change in the ratio of the sub-band area ratio 1668:1690 per $\mathrm{cm}$, which reflects non-reducible/reducible collagen crosslinks, ${ }^{27}$ with a substantial and significant decrease, about $30 \%$, of crosslinked collagen in the knock-out mice (Figure $2 \mathrm{a} ; P=0.04$ ). This reflects a decrease in to mature crosslinks in the knock out mice, which might have a serious 
a
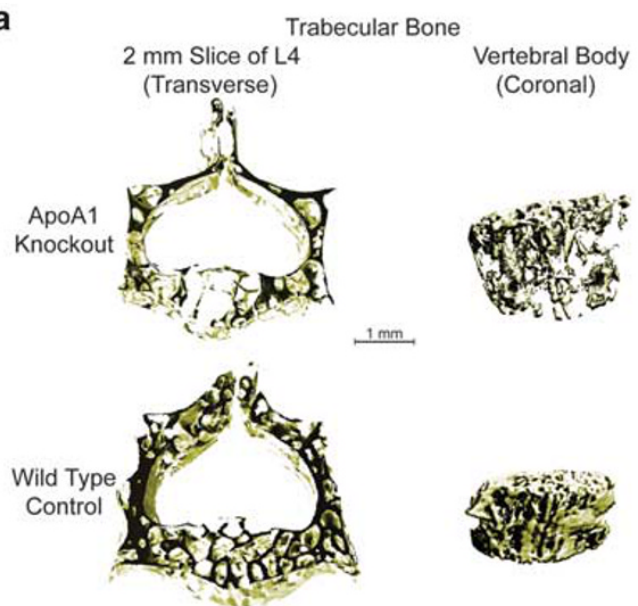

b
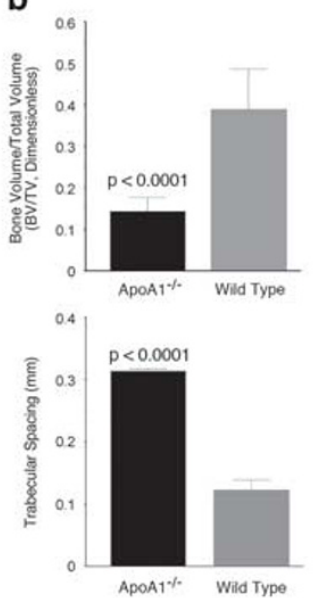
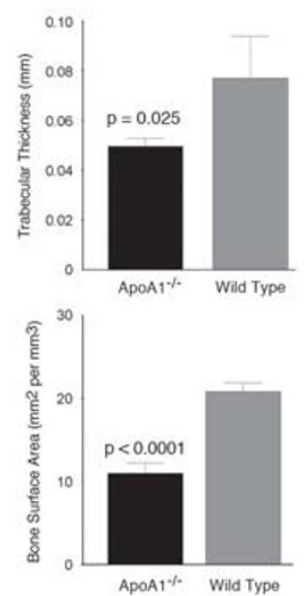

c
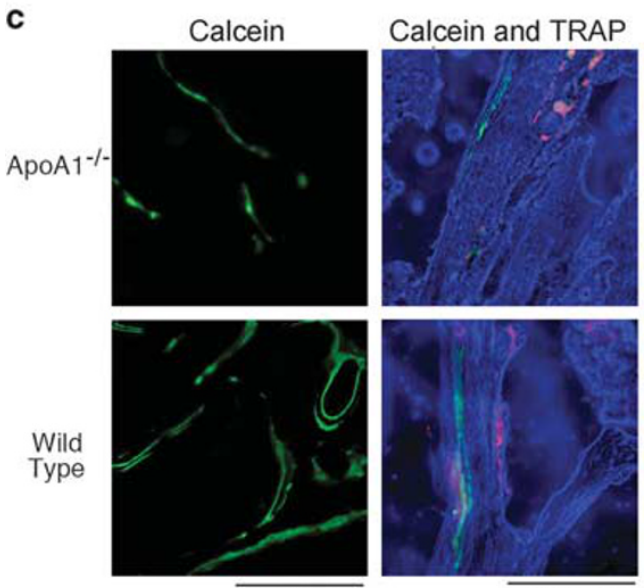

$250 \mu \mathrm{m}$

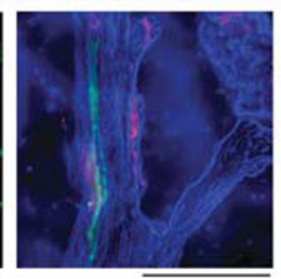

$150 \mu \mathrm{m}$
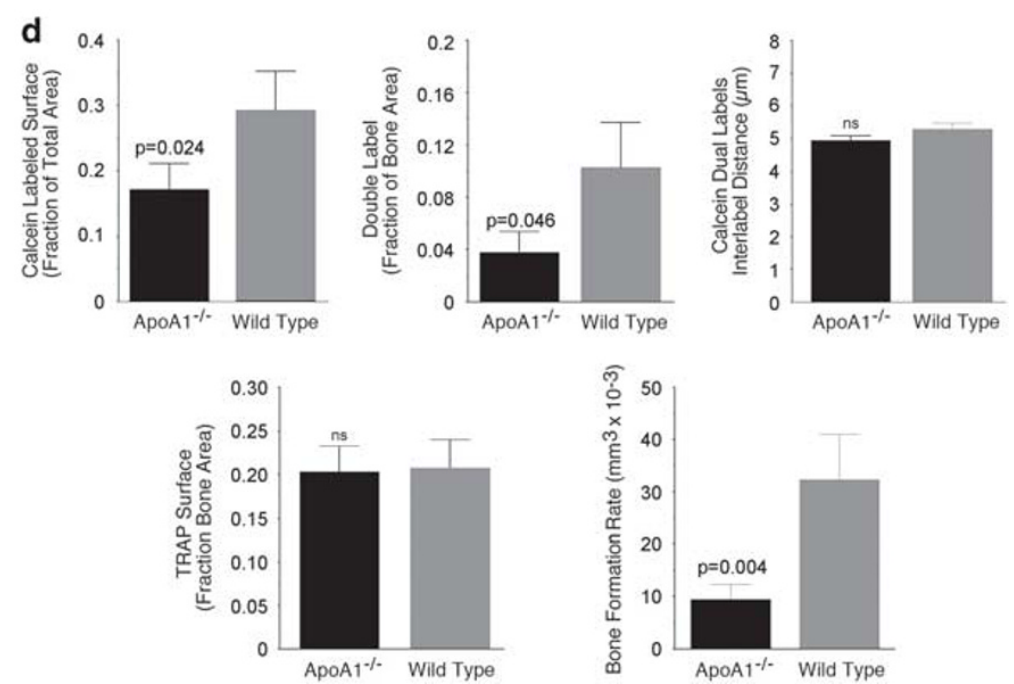

Figure 1 Reduced bone formation in apoA-1 $1^{-/-}$mice relative to WT. In this figure, $n=4$, mean \pm s.d.; comparisons used Student's $t$-test. (a) Micro-CT images as three-dimensional projections of $2 \mathrm{~mm}$ slices from lumbar vertebrae of apoA-1 ${ }^{-1-}$ and WT mice at 12 weeks of age. (b) Static parameters for four control and four knock-out mice as in (a). Trabecular volume per total bone volume (BV/TV), trabecular thickness (Tb.Th), trabecular spacing (Tb.Sp), and bone surface (BS) were significantly decreased in the apoA-1-deficient compared with the WT mice than in WT mice. (c) Calcein labels on lumbar vertebrae (green, left column) revealed that the apoA-1 $1^{-/-}$mice had decreased bone formation rate relative to their WT counterparts. On the contrary, TRAP labeling showed no differences in TRAP activity between the two groups (red, TRAP positive cells). (d) Summary results of dynamic histomorphometry by blinded observers. Note that the calcein-labeled surface, double labels, and bone formation rate were greatly decreased in the apoA-1 compared with the WT mice. In contrast, calcein labeling interval and osteoclast (TRAP) surface per total bone surface were similar between the examined groups.

impact on bone mechanics. A similar $\sim 30 \%$ reduction of collagen cross-linking was reported by Liu et al. ${ }^{28}$ for NHERF- $1^{-1-}$ relative to WT mice. To examine further how changes in collagen crosslinks reflect alterations in bone strength we tested the biomechanical properties of femora by three-point bending. Maximum load to fracture was reduced by $30 \%$ but not significantly due to variability $(P=0.52)$, bending strength decreased $40 \%$ with a trend, but not reaching significance $(P=0.1)$; modulus of elasticity was reduced $60 \%$, in keeping with reduced crosslinks, and this was significant $(P=0.02$; Figure $2 \mathrm{~b},(\mathrm{~A}-\mathrm{C}))$. Related findings included that, in addition to less trabecular bone, bones from apoA $-1^{-1-}$ mice had significantly increased bone marrow adipocytes in comparison with their WT counterparts (Figure 2c). This very interesting finding might point to a shift of MSC toward lipoblastic differentiation, and might be responsible for the reduced osteoblastic function observed in the apoA-1 $1^{-1-}$ mice. No differences in height were observed between the tested mouse groups.

\section{HDL Receptor Effects, ACTH and Cortisol Production}

As apoA-1-deficient mice have severely reduced HDL levels, we determined whether apoA-1 deficiency is associated with changes at the expression level of scarbl gene that codes for SR-B1 the main HDL receptor. ApoA-1-null mice had twofold elevated Scarb1 mRNA levels, (Figure 3a). To test 

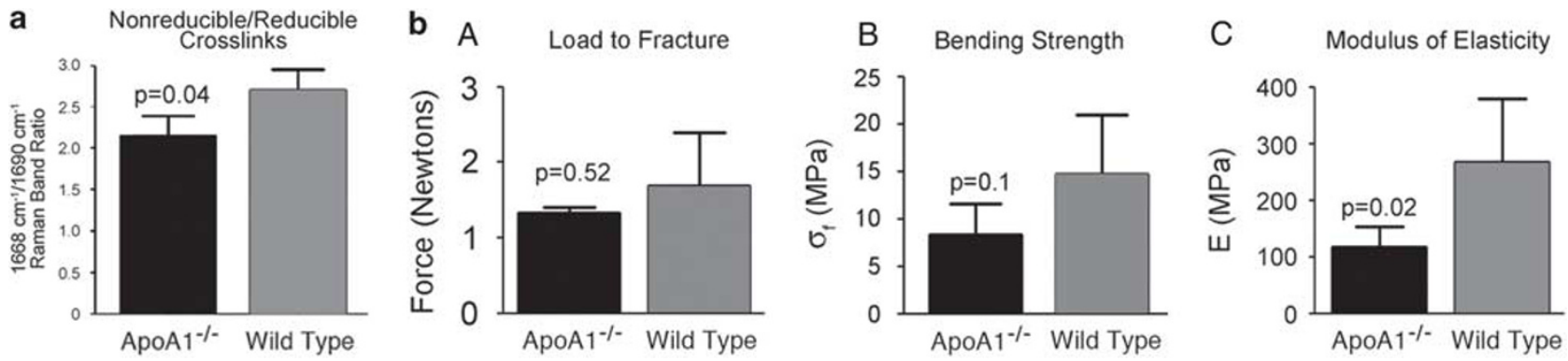

C
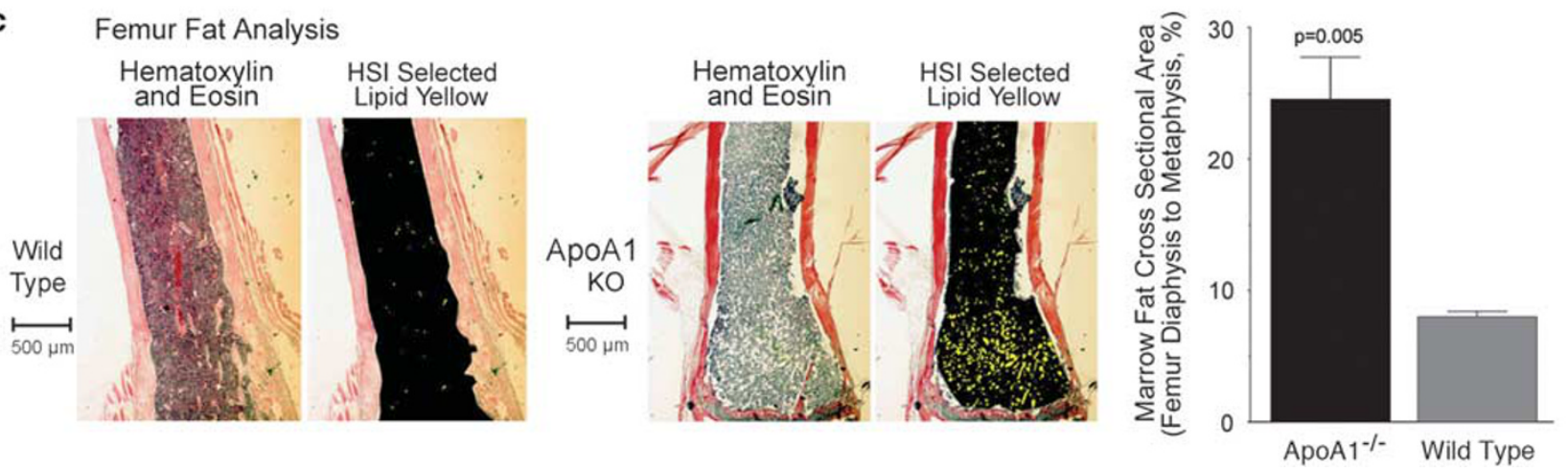

Figure 2 Bone quality and fat content in apoA-1-1- and WT mice. (a) Collagen cross-linking, by Raman Spectroscopy, revealed a substantial decrease (29\%) for the knock out compared with the WT mice. Statistical significance was considered at $P=0.04, n=5$. (b) Analysis of biomechanical properties of mice femora, using 3-point bending, showed that max load to fracture (A), bending resistance (B), and elasticity (C) were decreased in bone obtained from apoA-1 $1^{-1-}$ mice compared with WT bone. Only reduction in elasticity was significant $P=0.02$ due to variability, although trend in elasticity was seen $(P=0.10)$. (c) Histological and histomorphometric analysis revealed that the bone marrow of the apoA-1-deficient mice had significantly elevated adipocyte area in comparison with the WT group; the difference is highly significant in quantitative analysis of four femora $(n=4, P=0.004)$.
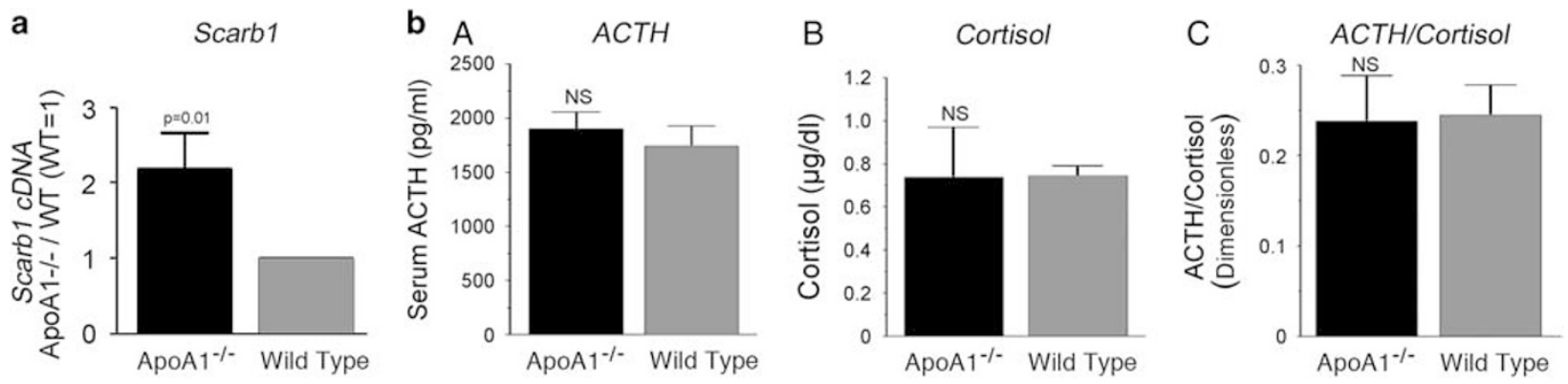

Figure 3 Scarb1, ACTH, and cortisol in apoA-1 knock-out and control mice. (a) Quantitative PCR analysis of Scarb1, in MSCs obtained from wild-type and knock out mice showed that apoA-1-deficient mice had twice-elevated Scarb1 mRNA levels relative to wild-type $(P=0.01)$. Mean \pm s.d., $n=5$. (b) Analysis of (A) ACTH, (B) cortisol, and (C) ACTH-to-cortisol ratio in serum from wild-type and apoA-1 ${ }^{-1-}$ mice showed no differences between the two groups. Data are normalized to the expression of GAPDH. All results are mean \pm s.d., $n=4$.

whether the effect of apoA-1 deficiency on bones is related to cortisol production we assessed the ACTH, cortisol and ACTH-to-cortisol ratio in plasma from apoA-1 $1^{-1-}$ and control mice. All of these were unchanged between the apoA-1-null and the WT mice (Figure 3b).

\section{ApoA-1 Deficiency Modifies MSC but not Osteoclast Differentiation, In vitro}

Our morphological and biomechanical experiments suggest that the reduced bone formation observed in the apoA-1- deficient mice most probably originates from an intrinsic defect of MSC to differentiate toward osteoblasts. To test this hypothesis we obtained MSC from the bone marrow of apoA- $1^{-1-}$ and WT mice and cultured them in osteogenic medium for 21 days (see the 'Methods' section). The conditions were identical, and included 10\% fetal bovine serum with normal lipoproteins. We observed greatly reduced ALP and bone mineral nodules (von Kossa) in MSC isolates from the apoA-1 $1^{-1-}$ mice compared with the controls $(P=0.04$ and $P=0.009$, respectively; Figures $4 \mathrm{a}$ and $\mathrm{b})$. 
a
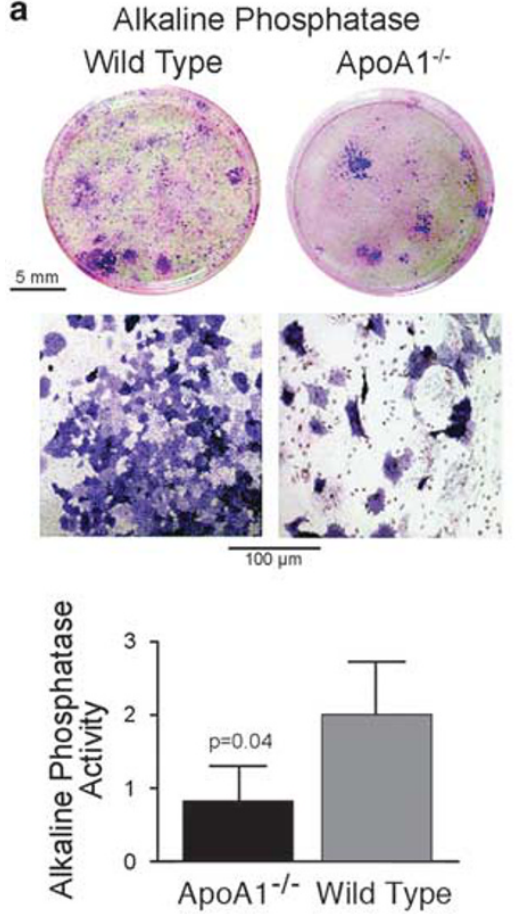

b
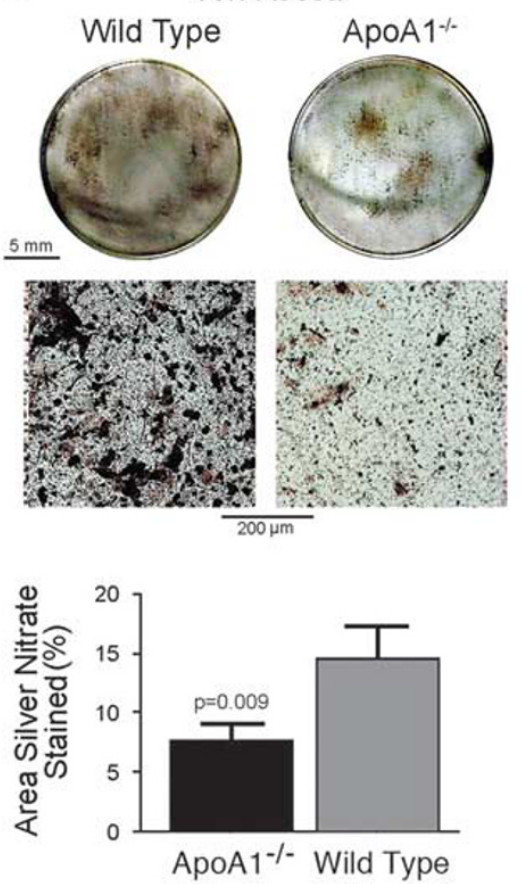

c
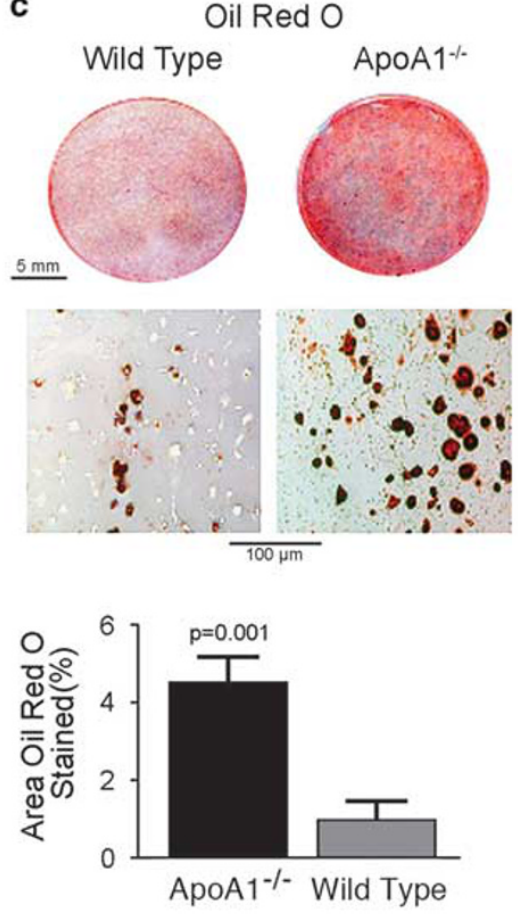

d TRAP in Monocytes Cultured with RANKL and CSF-1

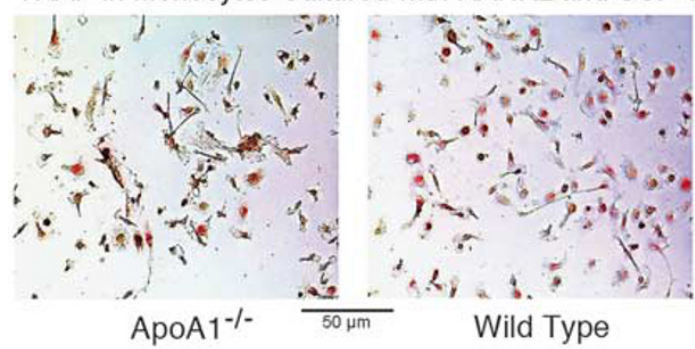

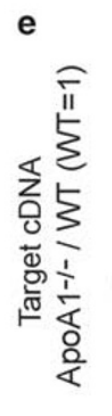

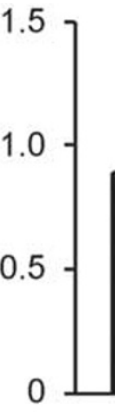

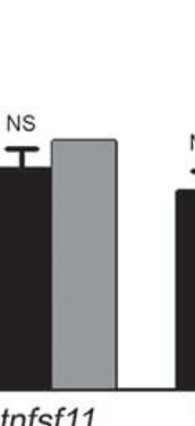

tnfsf11

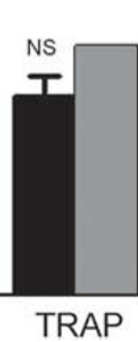

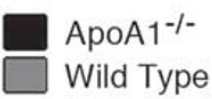

Figure 4 Effect of apoA-I deficiency on osteoblast and adipogenic differentiation in murine MSC. (a-c) Osteoblast and adipocyte differentiation of bone marrow-derived MSCs from wild-type and apoA-I mice. At 21 days after induction, alkaline phosphatase activity (ALP) (a), silver stain for mineral (von Kossa) (b), and oil red O stain for adipocytes (c) were performed. Top row: whole-culture dishes. Bottom row: photomicrographs of representative fields. Fields shown are $250 \mu \mathrm{m}$ in (a) and (c), $500 \mu \mathrm{m}$ in (b). Bar graphs summarizing densitometry of four assays are shown below the photomicrographs. The analysis was performed on images obtained from different areas of each dish under $\times 10$ magnification, with the use of Image $J$ software. ALP and mineral were significantly reduced $P=0.04$ and 0.009 , respectively; oil red $O$ was greatly augmented, $P=0.001$ in the apoA-1 $1^{-}$mice relative to control wild-type. (d) On the contrary, apoA-1 deficiency did not viably affect bone marrow monocyte differentiation toward osteoclasts. Bone marrow cells were collected from wild-type and apoA-1-deficient mice and cultured in M-CSF and RANKL to induce osteoclast differentiation. TRAP activity was labeled in cell cultures at day 14 showed no difference were between the two groups. (e) Real-time PCR analysis confirmed lack of difference in osteoclast differentiation. RANKL (Tnfsf11) Cathepsin K (Ctsk), TRAP (Trap). Each assay is expressed as fraction of the wild-type normalized to unity.

In stark contrast, Oil Red $\mathrm{O}$ stain revealed that the adipogenic capacity was prominent in the MSC from apoA-1 $1^{-I-}$ mice $(P=0.001)$, supporting a positive effect of apoA-1 deficiency on bone marrow fat formation (Figure 4c).

Consistent with normal TRAP area in apoA-1-deficient animals, osteoclast differentiation in vitro was unaffected by the knockout. Marrow cells from WT and apoA-1-deficient mice were cultured in M-CSF and RANKL to induce osteoclast differentiation. TRAP activity at day 14 was indistinguishable between the two groups (Figure 4d). ${ }^{29}$ Expression of mRNAs for TRAP (Acp5 gene), cathepsin K (Ctsk), and RANK (Tnfrsf11a), key osteoclast features, were not significantly different in WT and apoA-1 KO mice (Figure 4e). In aggregate these data show that apoA-1 deficiency suppresses differentiation of bone-forming osteoblasts, but has no significant effect on differentiation of bone resorbing osteoclasts.

\section{Analysis of Cell Signals Related to MSC Differentiation}

Pathway analysis of marrow cell mRNAs and proteins were then done, including key targets relating to MSC maintenance 
and differentiation. Main findings are shown in Figure 5. For additional studies not contributory to main mechanisms see Supplementary Table S3. In the marrow of $a p o A-1^{-1-}$ mice CXC chemokine ligand 12 (CXCL12) mRNA decreased threefold and CXC Receptor 4 (CXCR4) increased threefold, consistent with reduced signaling in a pathway that supports MSC homing and osteoblast generation (Figure 5a). The chemokine CXCL12 is regulated, in part by AnnexinA2, ${ }^{30}$ the mRNA of which was decreased significantly in the $\mathrm{KO}$. Consistent with increased committed adipoblasts in the overall MSC pool, PPAR- $\gamma$ mRNA was greatly increased, reflecting increased adipocytes and committed precursors (Figure 5a). In symphony with decreased committed osteoblasts in the MSC pool, in apoA-1 $1^{-/-}$animals, RUNX2, and collagen type 1a mRNA were decreased. The apoA-1 ${ }^{-1-}$ phenotype included increased CCAAT/enhancer-binding protein $\alpha$ (CEPBA) mRNA, which interacts with important cell proliferation and differentiation signals, and involvement in increased adipocyte differentiation. ${ }^{31}$ The intermediate protein NHERF-1 increased significantly and approximately twofold in the apoA-1 $1^{-1-}$ marrow. This was unexpected since, in osteoblasts, NHERF-1 regulates mineral accumulation and its absence is another factor that promotes adipogenesis. ${ }^{28}$ Because PPAR- $\gamma$ and RUNX2 are of central importance in adipocyte and osteoblast differentiation, respectively, the PCR results were confirmed by Western blotting and flow cytometric analysis (Figures $5 \mathrm{~b}$ and $\mathrm{c}$ ). Additional assays with interesting results but unclear meaning included analysis for ALP and apoA-2 in apoA-1 $1^{-1-}$ and WT marrow cells (Supplementary Table S3). ALP is a key enzyme in matrix production, and showed a trend to decreased expression in keeping with the result of decreased type I collagen mRNA, apoA-2, the second most abundant HDL lipoprotein, normally is $\sim 25 \%$ of the ApoA-1 concentration. ApoA-2 mRNA was almost twice as abundant in the knock-out mouse as in the WT, and showed a trend, but not significance due to variability.

\section{DISCUSSION}

In the present study we explored the effect of apoA-1 deficiency on bone in mice. Static histomorphometry revealed that the apoA-1-deficient mice have greatly reduced bone mass compared with the WT. This finding is in concert with the epidemiological studies linking reduced HDL with osteoporosis in humans. ${ }^{15}$ Dynamic histomorphometry demonstrated that apoA-1 $1^{-1-}$ mice had significantly reduced bone formation rate in comparison with their WT counterparts, but the osteoclast TRAP surface was unchanged. The reduction on bone mass in the $\mathrm{KO}$ mice was associated with altered biochemical composition and biomechanical properties. More specifically, Raman analysis indicated reduced collagen cross-linking and impaired mineralization that also occur senile osteoporosis and osteogenesis imperfecta. Bone elasticity was significantly reduced, in keeping with the collagen and mineral defects. Impaired bone quality can reflect reduced bone formation or augmented bone degradation.

It is well-established that SR-BI, is a high-affinity HDL receptor that mediates selective uptake of $\mathrm{HDL}-\mathrm{CE}$ (HDLderived cholesteryl ester) both in vitro and in vivo. Given that apoA-1 is a cardinal component of HDL biogenesis, in the present study we looked carefully for effects related to the SR-B1 high-affinity HDL receptor (Scarb1) in apoA-1-deficient mice. In Scarb1 knock-out mice, the adrenals are unable to derive normal amounts of cholesterol for steroid synthesis from HDL, and consequently have very high ACTH but normal cortisol due to feedback regulation. ${ }^{16,17}$ Here, the apoA-1-null mice had twofold elevated Scarb1 mRNA. This might represent a feedback induction of Scarb1 due to altered cholesterol transporter(s)-related promoter activity, which if so, is a novel phenomenon; however, further work will be needed to substantiate this hypothesis. On the other hand, careful controls for ACTH and glucocorticoids in plasma from apoA-1 ${ }^{-1-}$ and control mice showed entirely normal profiles, indicating that the low levels of HDL still allowed the adrenals to function in Scarb1 competent animals. Thus, differences in ACTH would not support bone formation and interfere with the apoA-1 knock-out effect. ApoA-2 is the second most abundant protein HDL protein in vertebrates, facilitating cholesterol efflux, and has been shown to increase in the absence of apoA-1;32 apoA-2 is involved in metabolic functions including plasma $\mathrm{HDL}$ maintenance, insulin resistance, and weight gain. That apoA-2 is retained may be reflected in the normal ACTH, and may affect the bone phenotype in undetermined ways. Our data, nevertheless, highlight the impact of cholesterol metabolic pathways on bone function. ${ }^{4,13}$ However, we found no effect on genes that directly regulate cholesterol synthesis and modification, namely Ggps, Fdps, Hmgcr, and Cptla. Notably, in apoA-1 $1^{-1-}$ cells, expression of apoA-2 was twice that seen in WT cells. This finding is in line with other studies ${ }^{32}$ and possibly reflects an adaptation to loss of apoA-1.

Further to investigate effects of apoA-1 deficiency in bone, we studied the differentiation of MSC from apoA-1 $1^{-/-}$and WT animals. MSC cultured in identical conditions including fetal bovine serum with normal lipoproteins, surprisingly, showed greatly reduced ALP and bone mineral nodules in MSC isolates from the apoA-1 $1^{-1-}$ mice. In contrast, adipogenic capacity was more prominent in MSC from apoA $-1^{-1-}$ mice, compared with the WT ones. These results suggest strongly that sub-populations of MSC are altered in the apoA-1-deficient animals and are in symphony with the recent concept, supported by the in vivo MSC fluorescent labeling that there are populations of MSC with differing probability of forming various types of cells, and that these populations are dynamically regulated. ${ }^{33,34}$ In parallel, decreased committed osteoblasts in the MSC pool in apoA$\mathrm{I}^{-/-}$animals displayed a remarkable reduction of RUNX2 and collagen type 1a mRNAs. Not surprisingly, other osteoblast product mRNAs, such as osterix and osteocalcin were decreased, even though they did not reach the level of 

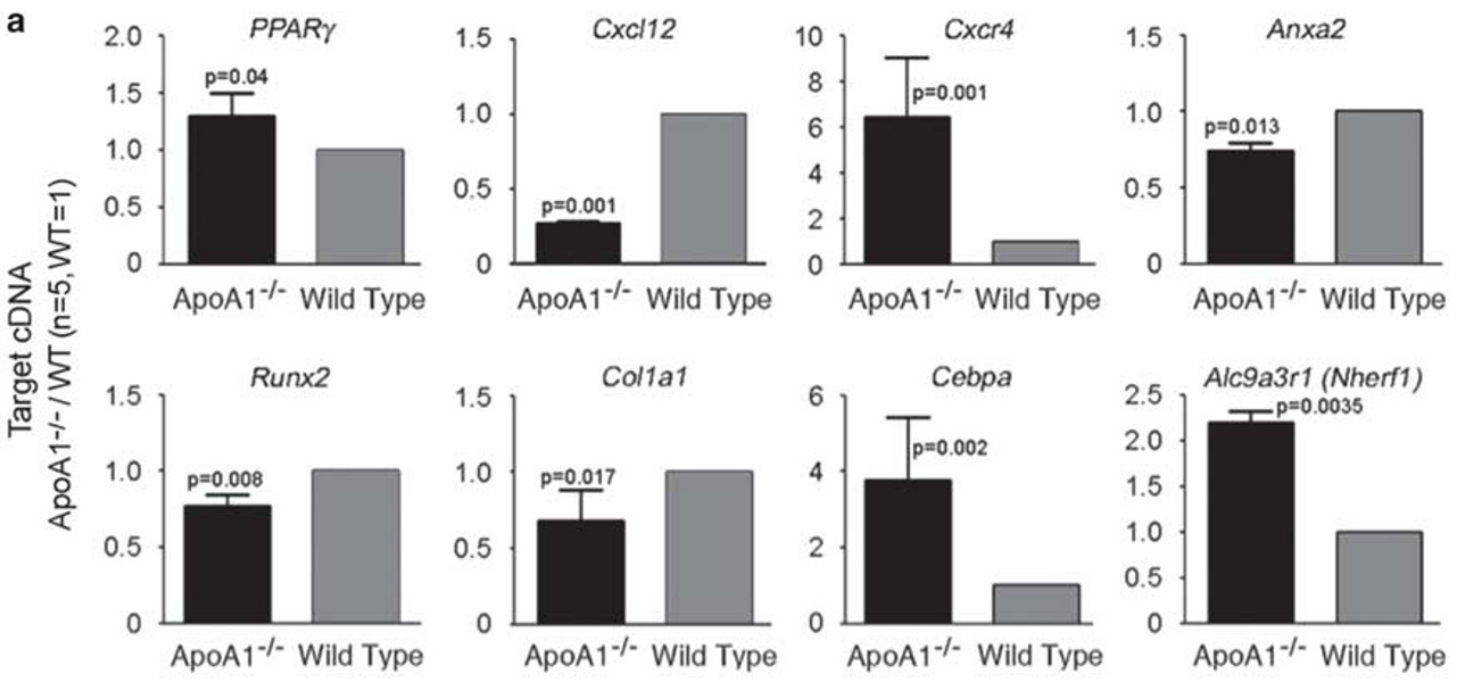

b

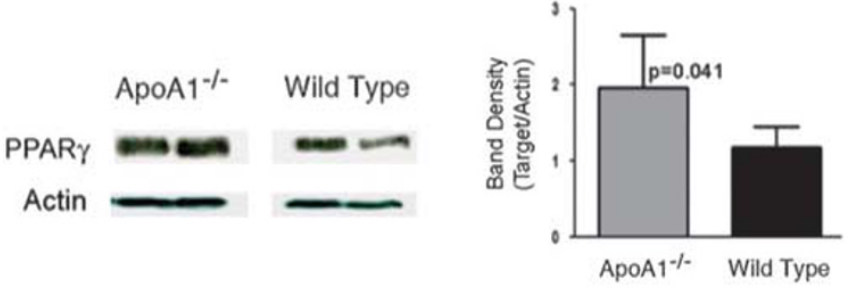

c
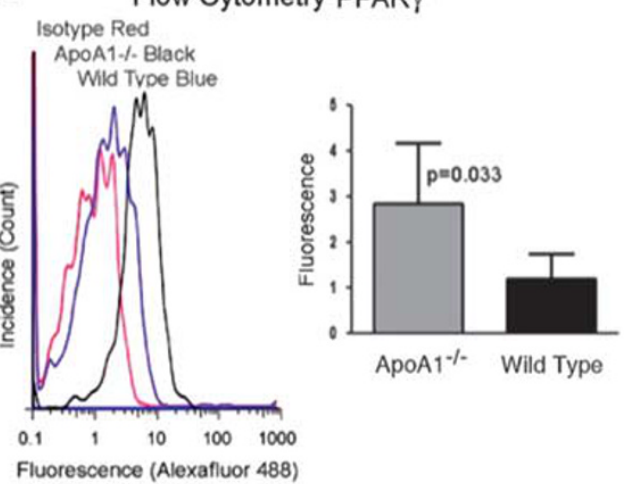

Flow Cytometry RUNX2

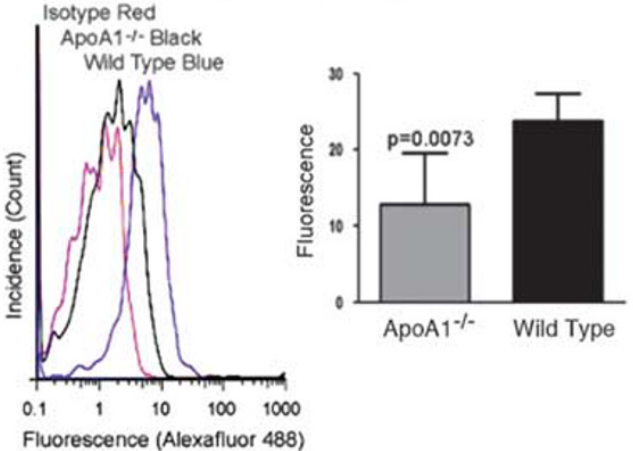

statistical significance. On the other hand, osteoclasts are derived from hematological precursors in different pools, and did not vary between the apoA-1 $1^{-1-}$ and WT animals. Another interesting finding of the present study was that the number of bone marrow adipocytes was significantly elevated in the apoA-1-deficient in comparison with the WT mice. Consistent with increased committed adipoblasts in the overall MSC pool, PPAR- $\gamma$ mRNA and protein were greatly increased in the $\mathrm{KO}$ mice. PPAR- $\gamma$ is a key intermediate downstream of CEPBA in a cascade that regulates adipocyte production, ${ }^{35}$ even though CEPBA also mediates other differentiation and proliferation effects. This may explain, at least in part, our notable and rather unexpected finding that CEPBA was increased in the apoA-1 $1^{-1-}$ cells. General MSC markers protein tyrosine phosphatase receptor $\mathrm{C}$ and stem cell antigen-1 were unaltered (Supplementary Table S3), in keeping with similar total stem cell populations in the knockout and WT, further supporting our hypothesis that pools with predilection to differentiate one way or the other indeed change. 
Figure 5 Secondary effects of apoA-1 deficiency on bone and MSC development. All graphs indicate mean $\pm s . d$. (a) Quantitative analysis of mRNAs important in MSC, adipocyte, and osteoblast differentiation. All studies here had $n=4$ for both ApoA-1 ${ }^{-/-}$and wild-type mRNA isolates. The mRNA levels of $C_{x C l 12}, C_{x c r}$, Anxa2, another regulator of stem cell homing to the osteoblast niche, were significantly reduced in the ApoA-1 ${ }^{-1-}$ animal relative to the wild-type. The osteoblast-specific genes Runx2 and type I collagen Col1a1, were significantly reduced in bone marrow cells from null mice. Runx2 increased $<25 \%$, and this is particularly important, so this was studied at the protein level, (b and c) below panels. In contrast, mRNA expression of the Cxcl12 receptor, Cxcr4, was elevated 6.5-fold in apoA-1-deficient mice. The key nuclear receptor regulator of lipoblast differentiation, PPARy was augmented significantly, but only $20 \%$ at the mRNA level in the apoA-1-deficient as compared with the wild-type C57BL/6 mice; this important receptor was also studied at the protein level (b and c, below panels). Two more noteworthy findings were that the CCAAT/enhancer-binding protein-a, cebpa was increased fourfold in the apoA-1-deficient mice; cebpa upregulation is a feature of osteoporosis in other contexts including glucocorticoid exposure. A surprising finding was a large increase in S/C9a3r1, the sodium-hydrogen exchange regulatory factor-1, which is expressed in osteoblasts during the mineral accumulation phase. (b) Expression of the important genes Ppary and RunX2, significantly but moderately increased and decreased, respectively, at the mRNA level, was studied by Western blotting. Examples of blots and actin reblots are shown on the left, with densitometry summarizing six wildtype and nine control lysates all normalized to actin on the right. These studies showed that Ppary increased $35 \%$, and Runx 2 decreased by half, in the apoA-1 $1^{-/-}$animals relative to the WT. (c) Expression of the important genes Ppary and Runx2 was confirmed using flow cytometric detection of the proteins. On the left are shown histograms of count and intensity. On the right are summaries of quantitative data on mean PPAR $y$ and Runx 2 antibody labeling from five isolates of each genotype in PPARy, or six WT and nine knock-out mice for Runx2. In parallel with our previous findings the protein levels of Runx2 were significantly reduced, while the protein levels of PPARy were greatly elevated in the apoA-1 KO as compared with the WT mice.

Other findings of interest, but fitting the central interpretation less well, included that the intermediate protein NHERF-1 increased significantly, approximately twofold, in the apoA-1 $1^{-1-}$ marrow. This interesting PDZ domain protein integrates function of several G-protein-coupled receptors including the PTH receptor with membrane transport proteins, ${ }^{36}$ which is required for normal osteoblast function and in its absence adipocyte differentiation increases. ${ }^{28}$ Hence, our finding might reflect that NHERF-1 is important in osteoblast terminal differentiation and its absence promotes adipocyte alternate differentiation, whereas when apoA-1 is present and osteoblast differentiation is disfavored, NHERF-1 might increase in partial compensation. Other late osteoblast mineralization genes the sodium/hydrogen exchangers NHE1 and 6, were invariant but markedly reduced in the NHERF-1-deficient animal. Thus, the increase in NHERF-1 is interesting but does not have consequences reciprocal to those of NHERF-1 knockout.

Another finding of our study is that CXCL12 mRNA was strongly suppressed in apoA-1 $1^{-1-}$ animals; on the contrary, the mRNA for its receptor, CXCR4, was significantly elevated. The CXCL12/CXCR4 axis is cardinal in MSC homing and bone synthesis and Cxcr4 deletion perturbs osteoblastogenesis and bone development. ${ }^{37-39}$ Therefore, our data most probably suggest that apoA-1 deficiency reduces the function of CXCL12/CXCR4 signaling axis with possible feedback compensation, firing the shift from osteoblast to adipocyte production. The chemokine CXCL12 is established to be regulated, in other marrow contexts, by ANXA2. ${ }^{30}$ Notably, in the present study we uncovered that ANXA2 levels were significantly suppressed in the bone marrow of the apoA-1 $\mathrm{KO}$ mice further supporting our hypothesis that apoA-1 deficiency affects the CXCL12/CXCR4 signaling.

Collectively, in the present study we show for the first time that apoA-1, and probably HDL, has a central role in regulation of bone remodeling and maintenance of bone quality. This is reflected by impaired osteoblast function and reduced bone synthesis in apoA- $\mathrm{I}^{-1-}$ animals. Osteoclast differentiation seems to be unaffected. ApoA-1 deficiency reduces the capacity of MSC to differentiate toward osteoblasts but promotes adipogenesis. The molecular mechanisms that underline these phenomena are largely unknown and warrant further investigation. However, our data provide evidence that disturbed ANXA2-CLCX12CXCR4 interactions, at least in part, link apoA-1 deficiency and abnormal bone quantity and quality in mice. In summary, our data indicate that apoA-1 is required for normal bone cell differentiation, with the mechanism, at least in part, balancing MSC populations and adipocyte or osteoblast precursor pools.

Supplementary Information accompanies the paper on the Laboratory Investigation website (http://www.laboratoryinvestigation.org)

\section{ACKNOWLEDGMENTS}

This work was supported in part by the Marie Curie Reintegration Grant (IRG), FP7-PEOPLE-RG-2009, \#256402-«apoeostearthritis»; the University of Patras, Faculty of Medicine KARATHODORI Research Grants D.155 and E.073; the "ARISTIA I" of Hellenic GSRT Grant 248; the Department of Veteran's Affairs Grant BX002490 and by National Institutes of Health (USA) grants AR055208, and AR065407. This research study is an activity of the research network OsteoNet (http://www.osteonet.gr), of the University of Patras.

\section{DISCLOSURE/CONFLICT OF INTEREST}

The authors declare no conflict of interest.

1. Marini F, Brandi ML. Pharmacogenetics of osteoporosis: what is the evidence? Curr Osteoporos Rep 2012;10:221-227.

2. Sobieszczańska M, Jonkisz J, Tabin $M$ et al. Osteoporosis: genetic determinants and relationship with cardiovascular disease. Adv Clin Exp Med 2013;22:119-124.

3. Dempster DW. Osteoporosis and the burden of osteoporosis-related fractures. Am J Manag Care 2011;17:S164-S169.

4. Kawai M, de Paula FJ, Rosen CJ. New insights into osteoporosis: the bone-fat connection. J Intern Med 2012;272:317-329.

5. Colaianni G, Brunetti G, Faienza MF et al. Osteoporosis and obesity: role of Wnt pathway in human and murine models. World J Orthop 2014;5: 242-246.

6. Yamauchi M, Yamaguchi T, Nawata $\mathrm{K}$ et al. Increased low-density lipoprotein cholesterol level is associated with non-vertebral fractures in postmenopausal women. Endocrine 2015;48:279-286. 
7. Lin YY, Chen CY, Chuang TY et al. Adiponectin receptor 1 regulates bone formation and osteoblast differentiation by GSK-3 $\beta / \beta$-catenin signaling in mice. Bone 2014;64:147-154.

8. Zhao LJ, Jiang H, Papasian CJ et al. Correlation of obesity and osteoporosis: effect of fat mass on the determination of osteoporosis. J Bone Miner Res 2008;23:17-29.

9. Takada I, Kouzmenko AP, Kato S. PPAR-gamma signaling crosstalk in mesenchymal stem cells. PPAR Res 2010;pii:341671.

10. Lecka-Czernik B. Marrow fat metabolism is linked to the systemic energy metabolism. Bone 2012;50:534-539.

11. Cao JJ. Effects of obesity on bone metabolism. J Orthop Surg Res 2011;6:30.

12. Fernández-Murga $M L$, Leonor $M$, Vinué $A$ et al. Impact of estrogens on atherosclerosis and bone in the apolipoprotein E-deficient mouse model. Menopause 2015;22:428-436.

13. Dieckmann M, Beil FT, Mueller B et al. Human apolipoprotein E isoforms differentially affect bone mass and turnover in vivo. J Bone Miner Res 2013;28:236-245.

14. Bartelt A, Beil FT, Schinke T et al. Apolipoprotein E-dependent inverse regulation of vertebral bone and adipose tissue mass in C57BI/6 mice: modulation by diet-induced obesity. Bone 2010:47:736-745.

15. Ackert-Bicknell CL. HDL cholesterol and bone mineral density: is there a genetic link? Bone 2012;50:525-533.

16. Martineau $C$, Kevorkova $O$, Brissette $L$ et al. Scavenger receptor class $B$, type I (Scarb1) deficiency promotes osteoblastogenesis but stunts terminal osteocyte differentiation. Physiol Rep 2014;2:pii:e12117.

17. Martineau $C$, Martin-Falstrault $L$, Brissette $L$ et al. The atherogenic Scarb1 null mouse model shows a high bone mass phenotype. Am J Physiol Endocrinol Metab 2014;306:E48-E57.

18. Zaidi $M$, Sun $L$, Robinson $L J$ et al. ACTH protects against glucocorticoidinduced osteonecrosis of bone. Proc Natl Acad Sci USA 2010;107: 8782-8787.

19. Zannis VI, Koukos G, Drosatos $\mathrm{K}$ et al. Discrete roles of apoA-I and apoE in the biogenesis of HDL species: lessons learned from gene transfer studies in different mouse models. Ann Med 2008;40:14-28.

20. Fisher EA, Feig JE, Hewing $B$ et al. High-density lipoprotein function, dysfunction, and reverse cholesterol transport. Arterioscler Thromb Vasc Biol 2012;32:2813-2820

21. Kunnen S, Van Eck M. Lecithin:cholesterol acyltransferase: old friend or foe in atherosclerosis? J Lipid Res 2012;53:1783-1799.

22. Lyssenko NN, Nickel M, Tang $C$ et al. Factors controlling nascent highdensity lipoprotein particle heterogeneity: ATP-binding cassette transporter A1 activity and cell lipid and apolipoprotein Al availability. FASEB J 2013;27:2880-2892.

23. Karavia EA, Papachristou DJ, Liopeta $K$ et al. Apolipoprotein $A-1$ modulates processes associated with diet-induced nonalcoholic fatty liver disease in mice. Mol Med 2012;18:901-912.

24. Karavia EA, Papachristou DJ, Kotsikogianni I et al. Lecithin/cholesterol acyltransferase modulates diet-induced hepatic deposition of triglycerides in mice. J Nutr Biochem 2013;24:567-577.
25. Triantaphyllidou IE, Kalyvioti E, Karavia EA et al. Perturbations in the $\mathrm{HDL}$ metabolic pathway predispose to the development of osteoarthritis in mice following long-term exposure to westerntype diet. Osteoarthritis Cartilage 2013;21:322-330.

26. Dempster DW, Compston JE, Drezner MK et al. Standardized nomenclature, symbols, and units for bone histomorphometry: a 2012 update of the report of the ASBMR Histomorphometry Nomenclature Committee. J Bone Miner Res 2012; 28:2-17.

27. Orkoula MG, Vardaki MZ, Kontoyannis CG. Study of bone matrix changes induced by osteoporosis in rat tibia using Raman spectroscopy. Vibrational Spectroscopy 2012;63:404-408.

28. Liu L, Alonso $\mathrm{V}$, Guo $\mathrm{L}$ et al. $\mathrm{Na}+/ \mathrm{H}+$ exchanger regulatory factor 1 (NHERF1) directly regulates osteogenesis. J Biol Chem 2012;287: 43312-43321.

29. Robinson LJ, Yaroslavskiy BB, Griswold RD et al. Estrogen inhibits RANKL-stimulated osteoclastic differentiation of human monocytes through estrogen and RANKL-regulated interaction of estrogen receptor-alpha with BCAR1 and Traf6. Exp Cell Res 2009;315: 1287-12301.

30. Jung $Y$, Shiozawa $Y$, Wang $J$ et al. Annexin-2 is a regulator of stromal cell-derived factor-1/CXCL12 function in the hematopoietic stem cell endosteal niche. Exp Hematol 2001;39:151-166.

31. Li J, Zhang N, Xu J et al. Dexamethasone shifts bone marrow stromal cells from osteoblasts to adipocytes by C/EBPalpha promoter methylation. Cell Death Dis 2013;4:e832.

32. Wang $Y$, Sawashita W, Qian J et al. ApoA-I deficiency in mice is associated with redistribution of apoA-II and aggravated AApoAll amyloidosis. J Lipid Res 2011;52:1461-1470.

33. Zaidi M, Sun L, Blair HC. Special stem cells for bone. Cell Stem Cell 2012;10:233-234.

34. Park D, Spencer JA, Koh Bl et al. Endogenous bone marrow MSCs are dynamic, fate-restricted participants in bone maintenance and regeneration. Cell Stem Cell 2012;10:259-272.

35. Shi XM, Blair HC, Yang $X$ et al. Tandem repeat of C/EBP binding sites mediates PPARgamma2 gene transcription in glucocorticoid-induced adipocyte differentiation. J Cell Biochem 2000;76:518-527.

36. Weinman EJ, Hall RA, Friedman PA et al. The association of NHERF adaptor proteins with $\mathrm{G}$ protein-coupled receptors and receptor tyrosine kinases. Annu Rev Physiol 2006;68:491-505.

37. Liu C, Weng Y, Yuan $T$ et al. CXCL12/CXCR4 signal axis plays an important role in mediating bone morphogenetic protein 9-induced osteogenic differentiation of mesenchymal stem cells. Int J Med Sci 2013;10:1181-1192.

38. Shahnazari M, Chu V, Wronski TJ et al. CXCL12/CXCR4 signaling in the osteoblast regulates the mesenchymal stem cell and osteoclast lineage populations. FASEB J 2013;27:3505-3513.

39. Zhu W, Liang G, Huang $Z$ et al. Conditional inactivation of the CXCR4 receptor in osteoprecursors reduces postnatal bone formation due to impaired osteoblast development. J Biol Chem 2011;286: 26794-26805 